\title{
The circulating level of Non-coding RNA for breast cancer diagnosis
}

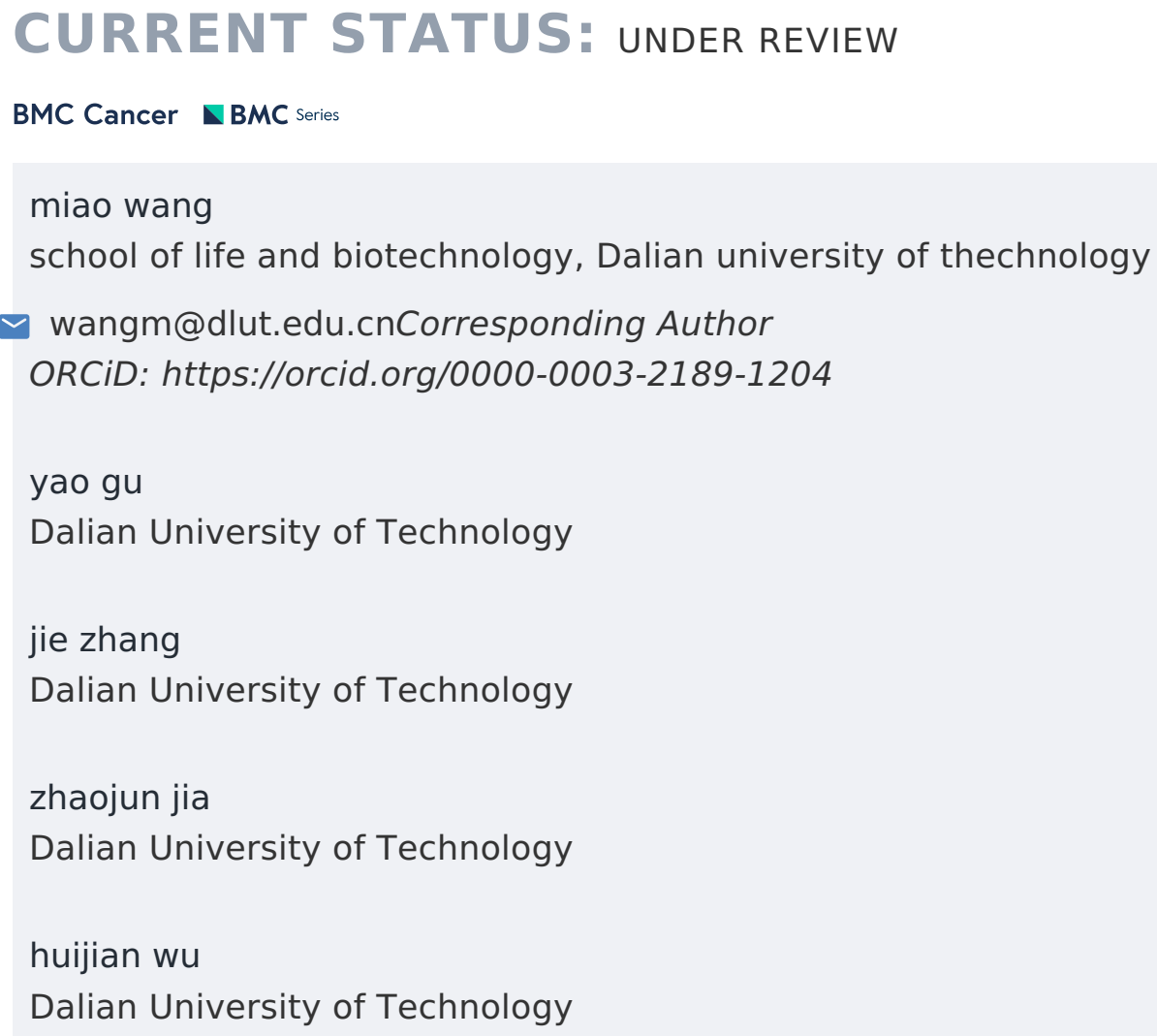

\section{DOI:}

10.21203/rs.2.19596/v1

\section{SUBJECT AREAS}

Oncology Cancer Biology

\section{KEYWORDS}

non-coding RNA, breast cancer, diagnosis 
Abstract

Background: Lots of non-coding RNAs expression affected breast cancer progression. However, there were no systemic analysis of non-coding RNAs for breast cancer diagnosis in circulatory system. Herein, we aimed to collect all the evidence to test the potential role of non-coding RNAs as novel biomarker in human breast cancers.

Methods: A comprehensive search strategy was used to search relevant literatures in the Web of Science, PubMed, and Embase databases from 2012 to November 2019. The correlation between noncoding RNAs in serum, plasma or blood expression and the diagnostic accuracy of BC markers were analyzed. The methodological quality of each study was assessed using the QUADAS-2. Statistical analysis was used the STATA (version 12.0), Meta-Disc1.4 and Review Manager (version 5.3) software. Results: The present meta-analysis the non-coding RNAs expression data of BC patients and healthy specimens' bloodfrom 2392 patients in 24 publications (32 studies). The pooled sensitivity, specificity, and AUC values were $0.82,0.83$, and 0.89 , respectively. Subgroup analyses showed that the expression of non-coding RNA (miRNA, circRNA, andIncRNA) in circulating (including blood, plasma, and serum) of BC was more prone to be detected in TNM stage and subtype of BC group, with a high value of the sensitivity and AUC.

Conclusions: The circulating (including blood, plasma, and serum) level of miRNAs, circRNAs, and IncRNAs for breast cancer diagnosis may be effective, particularly in TNM stage, subtype of BC (ER/PgR, PR or HER2/C-erbB-2) breast cancer. Further prospective studies on the diagnostic value of non-coding RNAs from breast cancer are needed in the future.

Full Text

Due to technical limitations, full-text HTML conversion of this manuscript could not be completed. However, the manuscript and Tables 1-5 can be downloaded and accessed as PDF files.

Figures 


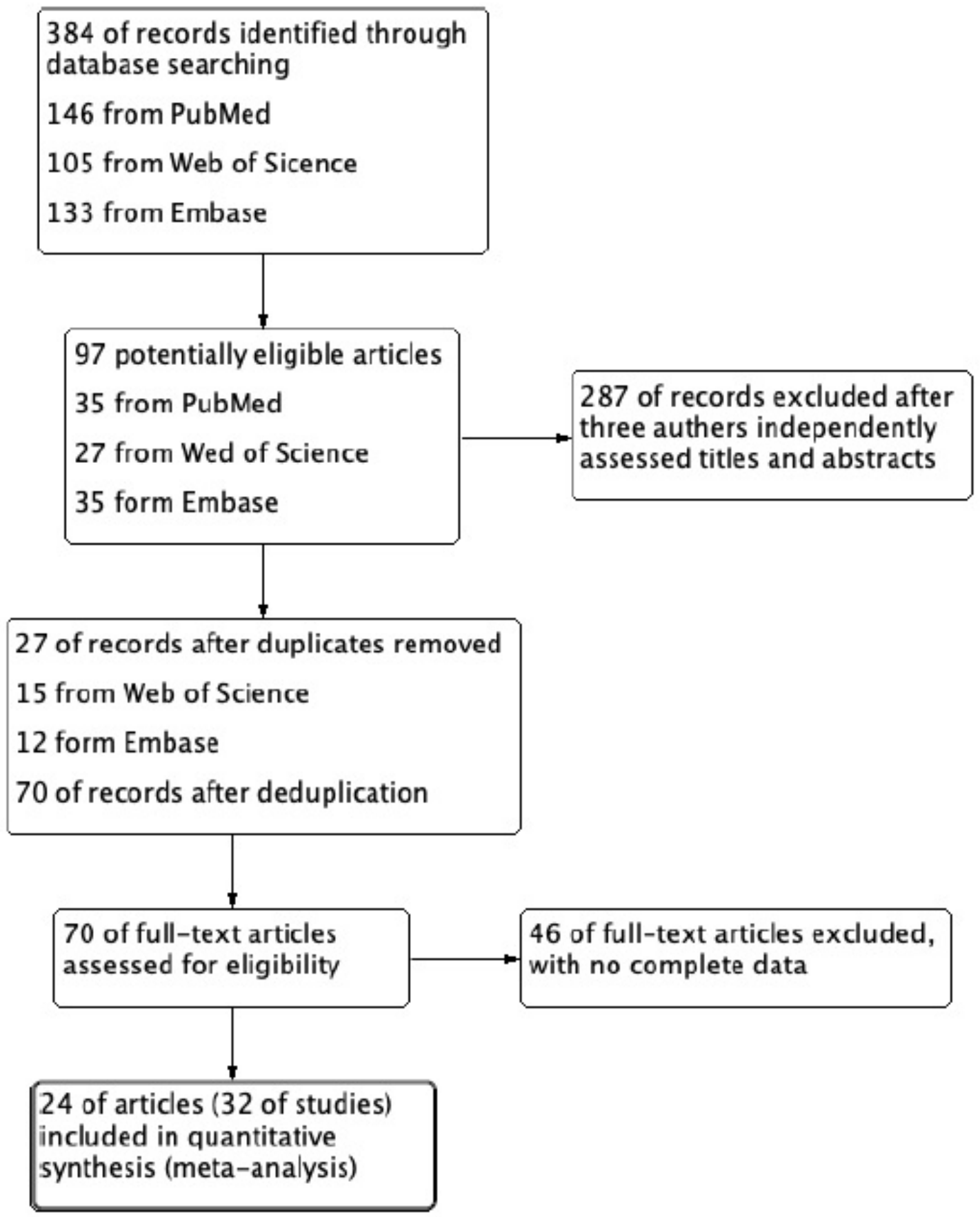

Figure 1

Flowchart of inclusion studies. 
A

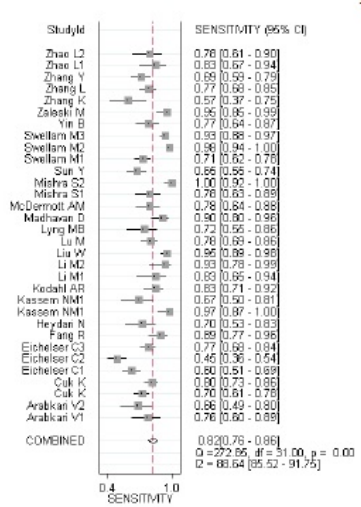

E

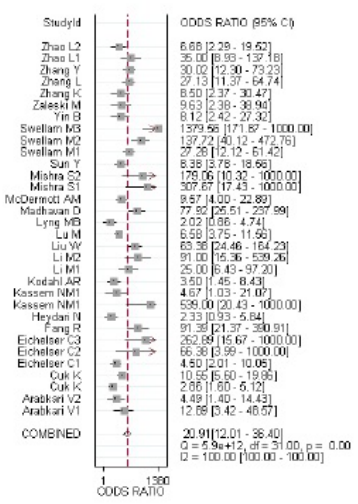

B

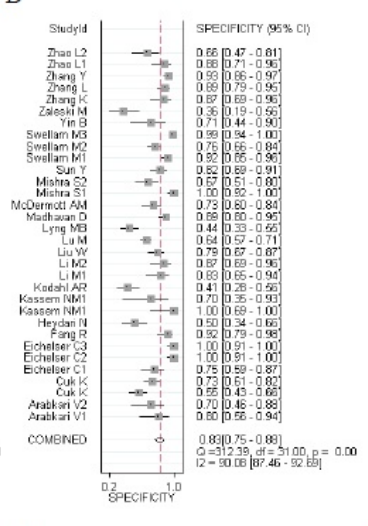

F

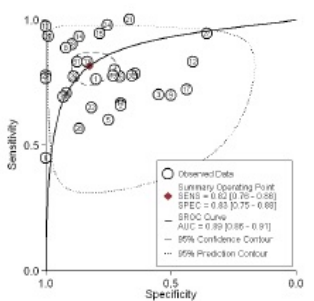

$\mathrm{C}$

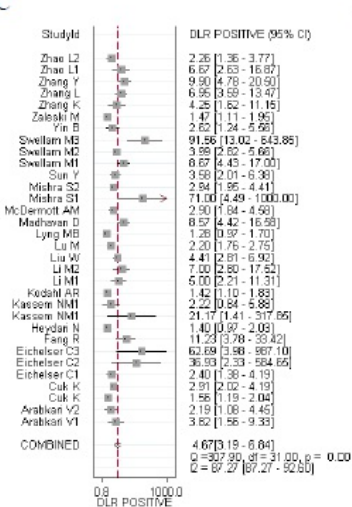

G

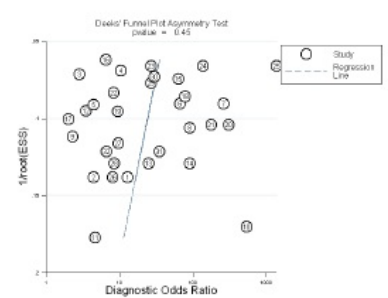

D

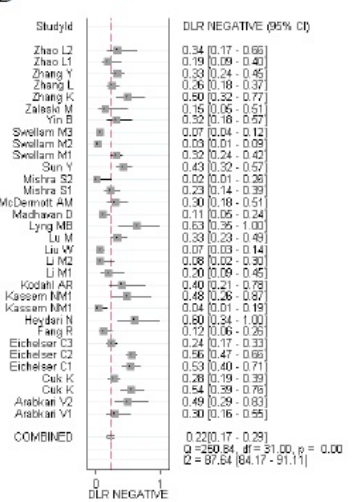

H

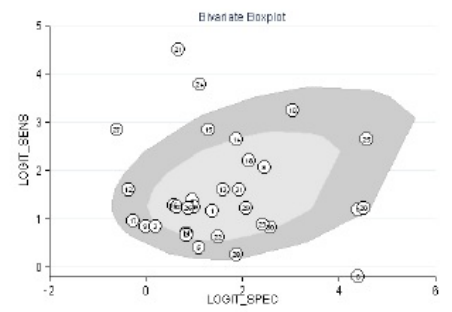

Figure 2

The results of meta-analysis in all studies for cancer diagnosis. The forest plots of (A) SEN, (B) SPE, (C) PLR, (D) NLR, (E)DOR, and (F) AUC. (G) Funnel plot to evaluate publication bias. (H)Bivariate boxplot to detected the heterogeneity. 


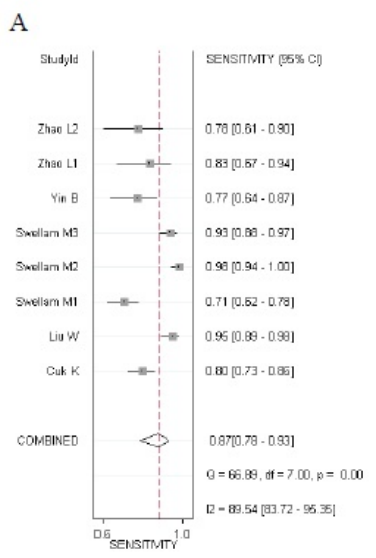

$\mathrm{E}$

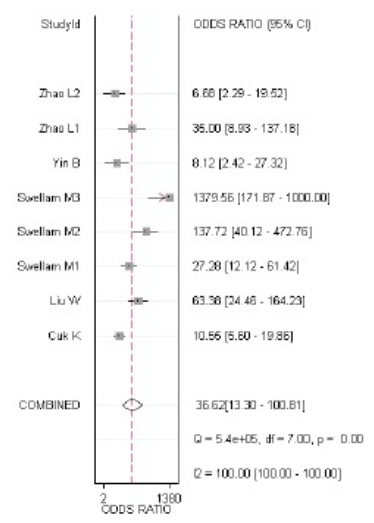

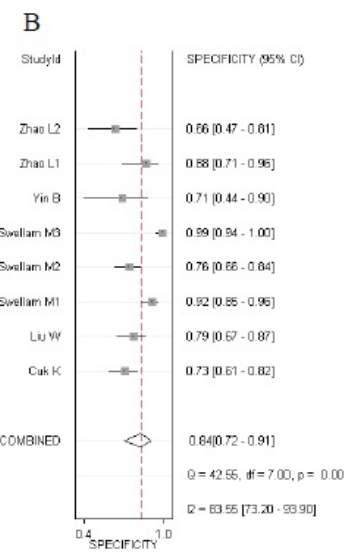

$\mathrm{F}$

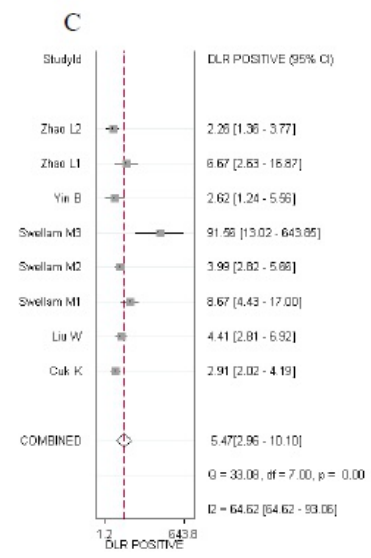

G
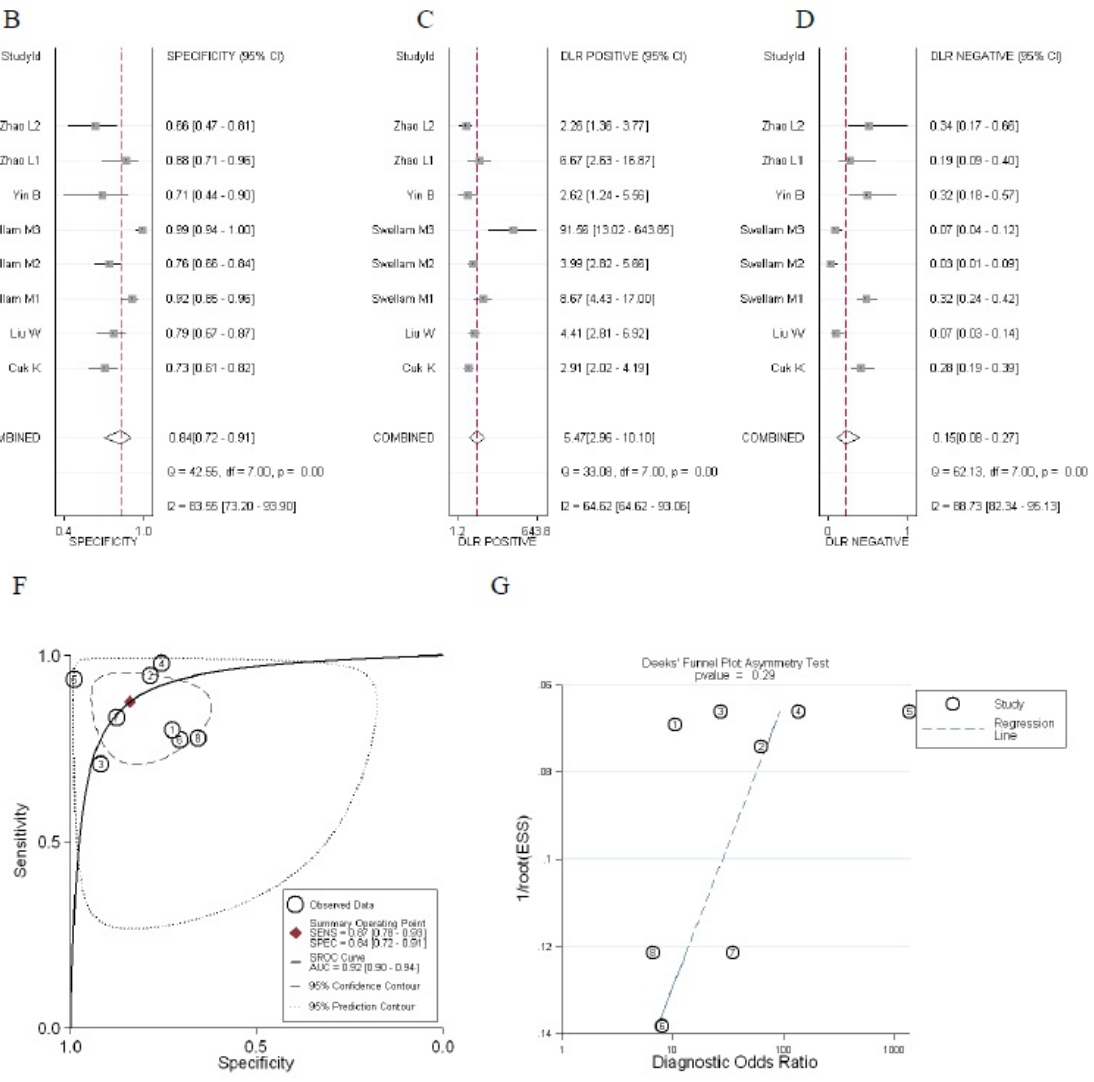

Figure 3

The results of meta-analysis in subgroup of the studies for non-coding RNA association with TNM stage. The forest plots of (A) SEN, (B) SPE, (C) PLR, (D) NLR, (E)DOR, and (F) AUC. (G) Funnel plot to evaluate publication bias. 
A

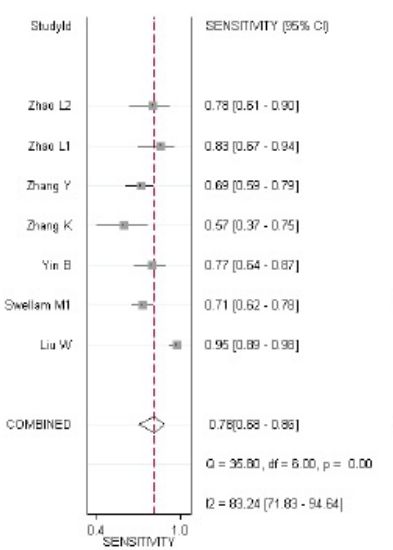

$\mathrm{E}$

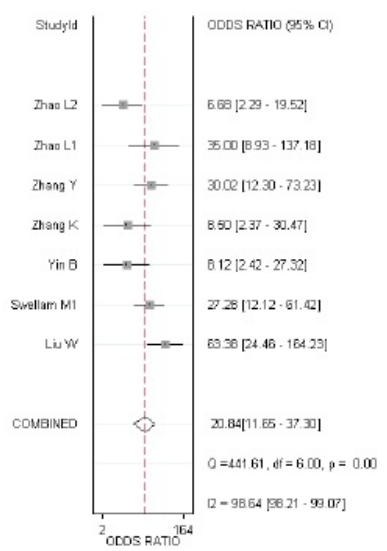

B

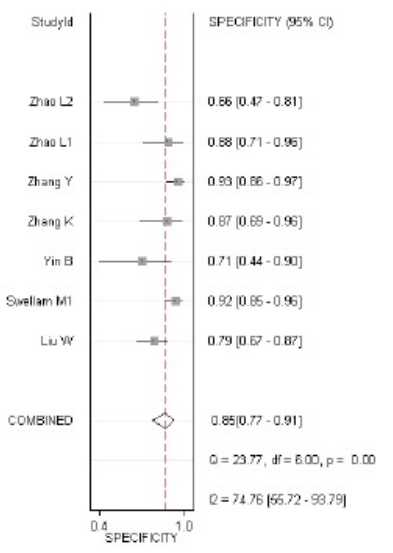

$\mathrm{F}$

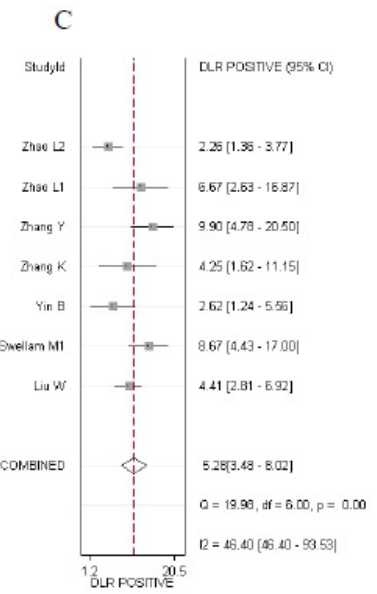

G
$\mathrm{D}$

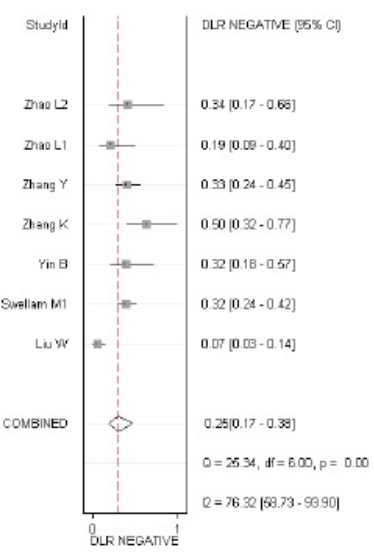

TR NEQGATV
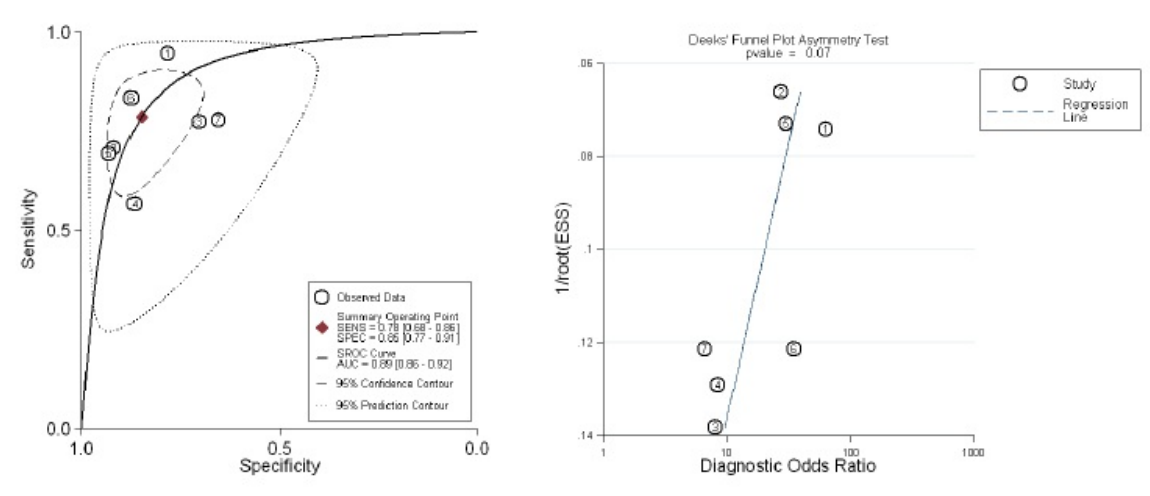

Figure 4

The results of meta-analysis in subgroup of the studies for non-coding RNA association with invasion. The forest plots of (A) SEN, (B) SPE, (C) PLR, (D) NLR, (E)DOR, and (F) AUC. (G) Funnel plot to evaluate publication bias. 


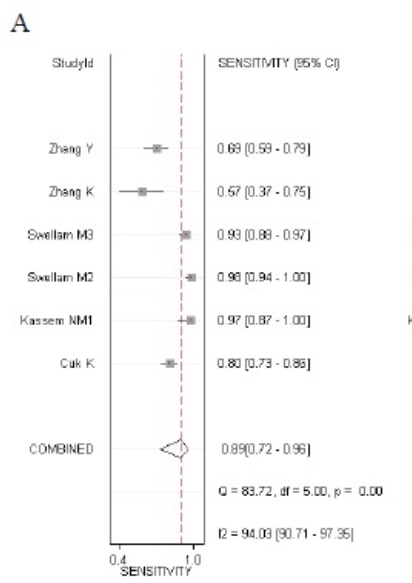

E

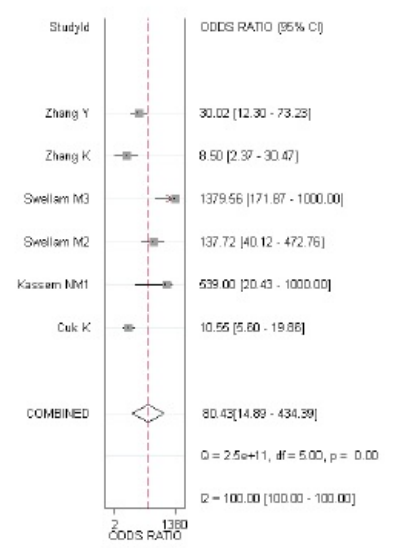

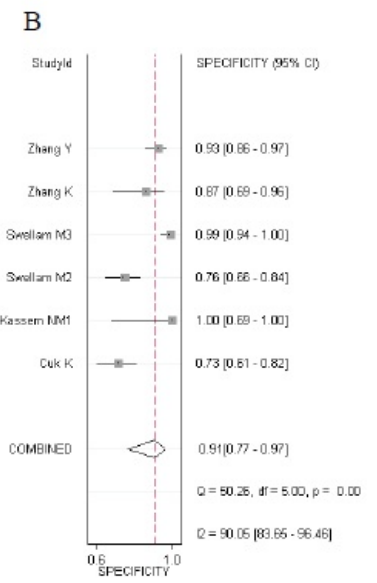

F

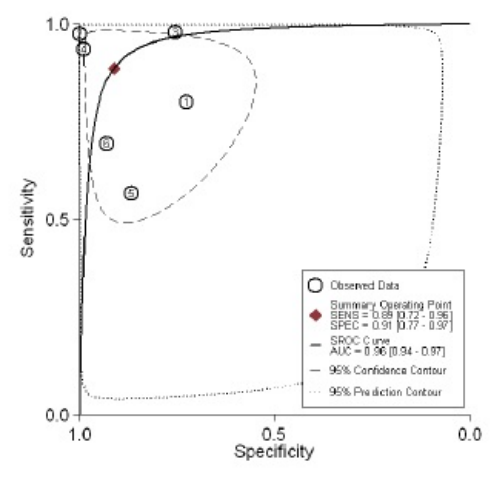

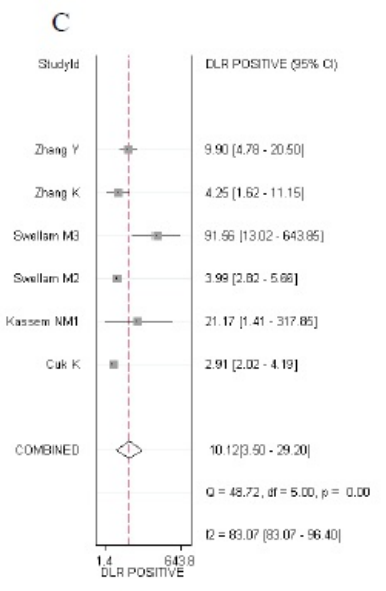

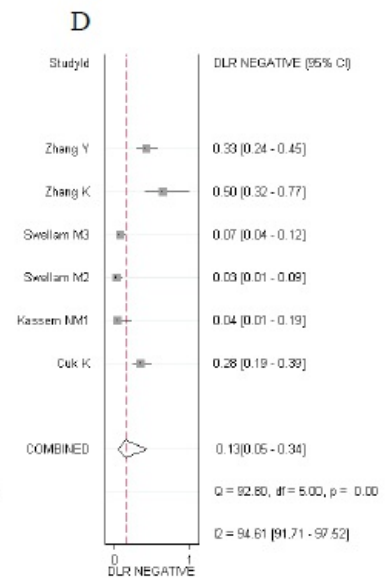

$\mathrm{G}$

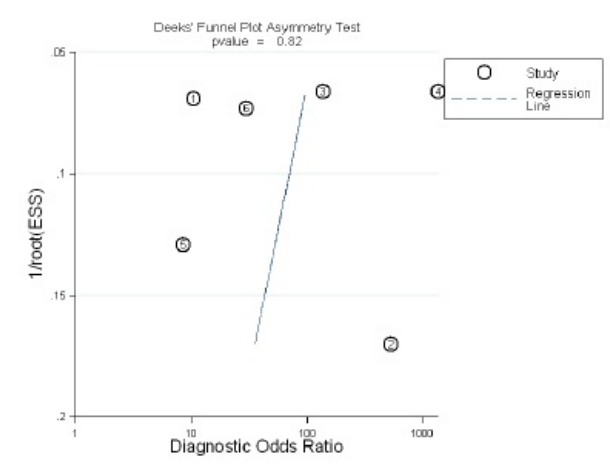

Figure 5

The results of meta-analysis in subgroup of the studies for non-coding RNA association with subtype of BC. The forest plots of (A) SEN, (B) SPE, (C) PLR, (D) NLR, (E)DOR, and (F) AUC. (G) Funnel plot to evaluate publication bias.

\section{Supplementary Files}

This is a list of supplementary files associated with this preprint. Click to download.

table s1 PRISMA 2009 Checklist.doc

sup1.pdf

Table1-5.pdf

Table S2 All characteristics of all studies.docx 